\title{
Caracterización del gesto deportivo patada de balón en movimiento en el fútbol sala. Estudio de caso
}

\author{
Laura Elizabeth Castro Jiménez* \\ Yeimy Damaris Leguizamón Torres** \\ Jenny Patricia Nieto Guevara*** \\ Kelly Johana Pabón Rodríguez ${ }^{* * * *}$
}

Recibido: 18 de junio de 2013 • Evaluado: 10 de julio de 2013

Aceptado: 25 de agosto de 2013

\section{Resumen}

En los últimos años, se ha extendido la práctica deportiva en personas en condición de discapacidad, pero existen muy pocos estudios sobre la descripción cinemática de los gestos deportivos como herramienta para optimizar la técnica y así conseguir mejores marcas a nivel nacional o mundial; de allí que el objetivo del estudio fue caracterizar cinemáticamente la patada de balón en movimiento en un deportista de alto nivel competitivo del fútbol sala con limitación visual. El estudio es descriptivo, tipo estudio de caso. Se hicieron diez capturas del gesto deportivo, caracterizando la biomecánica de la técnica del deportista, evaluando desplazamiento articular, velocidad y centro de gravedad; los resultados se contrastaron con una evaluación fisioterapéutica en la que se incluyó postura, flexibilidad y fuerza muscular. Se concluyó que el deportista no realizaba de forma adecuada el gesto deportivo, porque no

*Fisioterapeuta, Universidad Nacional; magíster en Salud Pública, Universidad Nacional; estudiante del Doctorado en Humanidades, Humanismo y Persona. Docente de la Facultad de Cultura Física, Deporte y Recreación; grupo de investigación Cuerpo, Sujeto y Educación, Universidad Santo Tomás, Bogotá.

"* Fisioterapeuta, Universidad Manuela Beltrán.

*** Fisioterapeuta, Universidad Manuela Beltrán.

"****Fisioterapeuta, Universidad Manuela Beltrán. Contacto: kellybmx18@hotmail.com 
alcanza los arcos de movimiento esperados durante la fase de pateo con balón quieto, lo que lleva a una disminución de la velocidad del gesto y de la potencia del pateo.

Palabras clave: Biomecánica deportiva, fútbol sala, desplazamiento articular, velocidad, centro de gravedad, discapacidad visual. 


\title{
Characterization of kick sporting gesture moving ball in futsal. Case study
}

\begin{abstract}
In recent years the practice has spread sports persons disability status, but there are few studies on the kinematic description of gestures sports as a tool to improve the technique and thus achieve better marks nationally or globally. That is why the aim of this study is to characterize kinematically's kick ball moving in a highly competitive sport of futsal visually impaired. The study is descriptive, a case study, where there were ten catches sporting gesture between which was chosen the best, which was performed with the biomechanical characterization technique athlete; assessing articular displacement, velocity and center of gravity these results were compared with an assessment that included physiotherapy in posture, flexibility and muscle strength. The conclusion was that the athlete was not performing adequately the sporting gesture, because not enough range of motion expected during the balloon still kicking and this leads to a decrease in the speed of the gesture and the power kicked.
\end{abstract}

Keywords: Sports biomechanics, futsal, joint displacement, velocity, center of gravity, visually disabled. 


\section{Introducción}

En la actualidad, la discapacidad no es vista como una limitación, sino como una deficiencia para hacer algunas actividades específicas. Esta condición, como consecuencia de accidentes, enfermedades, guerras, entre otros eventos, ya no se ve como problema sino como oportunidad para salir avante. Es así como el deporte se presenta como una alternativa no solo para superar la discapacidad, sino también para formarse profesionalmente.

Las personas en condición de discapacidad no pueden practicar un deporte ni competir en iguales condiciones que las personas sin discapacidad; por lo tanto, se ha creado una categoría o modalidad que se conoce como 'deporte adaptado' o 'deporte especial' (Campos, 2010; Castro, 2010). El deporte paralímpico estimula, desarrolla, mejora y fortalece las habilidades, destrezas y aptitudes psicomotrices, técnico-tácticas, físicas, cognitivas, sociales y afectivas para un buen desempeño integral de personas con discapacidad física, sensorial, y cognitiva (Secretaría de Cultura, Recreación y Deporte, 2003).

Entre los deportes para la población en condición de discapacidad se encuentra el fútbol sala para invidentes, en el que los jugadores desarrollan la habilidad para manejar el balón, lo que requiere una técnica individual de localización del sonido (balón con cascabeles) y del obstáculo -los jugadores rivales-. Todo esto se trabaja desde la orientación individual y colectiva, y el dominio espacial y corporal, que permite una coordinación para el regate, el pase a un compañero o el disparo a puerta, siempre intentando no perder el orden táctico individual y colectivo (Campos, 2010; Molina, 2006).

En Colombia, se ha reconocido que el deporte permite no solo rehabilitarse y desarrollar capacidades y aptitudes deportivas, sino también avanzar en el plano personal y profesional. Por tal razón, es importante ampliar la información acerca del deporte paralímpico y cómo perfeccionar la técnica y el gesto deportivo por medio del conocimiento de la biomecánica del deporte.

Como lo menciona Castro (2010), es necesario comprender cómo las personas en condición de discapacidad realizan los movimientos propios de la ejecución deportiva, lo cual se ha convertido en una necesidad en los últimos años y en un área con poco desarrollo investigativo. Por 
consiguiente, la investigación se centra en la caracterización de los principales gestos deportivos desarrollados en el fútbol sala como el pateo con balón en movimiento. A partir de allí, se pretende desarrollar un modelo biomecánico que analice la cinemática de los gestos más importantes.

Aunque existen varias formas de hacer una aproximación a la caracterización cinemática de los deportistas en condición de discapacidad, varios autores han descrito que la tecnología digital se convierte en una herramienta para el análisis del movimiento. Esto favorece la valoración fiable y rápida de los parámetros cinemáticos que describan el movimiento, permite caracterizar la técnica deportiva y, a partir de allí, ofrece herramientas para mejorarla y evitar lesiones por exceder ciertos rangos de movimiento al efectuar técnicas incorrectas.

\section{Materiales y métodos}

La presente investigación tuvo un alcance descriptivo y responde a un estudio de caso. Para ello, se evaluó un deportista de alto nivel competitivo del fútbol sala con limitación visual. Se hicieron diez capturas para el gesto deportivo de patada de balón en movimiento y se escogió la mejor, a partir de la cual se hizo la caracterización biomecánica de la técnica del deportista.

\section{A. Infraestructura y equipos}

El laboratorio utilizado para este estudio cuenta con la tecnología BTS ${ }^{\circledR}$ y se compone de: cámaras infrarrojas optoelectrónicas de alta velocidad que detectan la trayectoria de los marcadores colocados en el deportista; cámaras de video que captan el movimiento; un centro de control BTS Elite ${ }^{\circledast}$ que gestiona el sistema, analiza y elabora los datos adquiridos, y la unidad de proceso BTS Elite ${ }^{\circledR}$, que integra y sincroniza todas las señales de los dispositivos conectados.

El área de adquisición del laboratorio consta de una pista que mide $4 \mathrm{~m}$ de largo por 1,20 m de ancho, donde se lleva a cabo el registro de los movimientos del deportista. Presenta condiciones de iluminación especiales para obtener una buena imagen en la captura de los videos de movimiento 
y también para el registro de marcadores reflectivos del análisis cinemático. La técnica de captura se hace por medio de un sistema optoelectrónico, con seis cámaras digitales infrarrojas que capturan 1.000 imágenes por segundo y que cuenta, además, con cámaras digitales de video a color.

\section{B. Sistema de captura}

Se utilizan los programas diseñados por los fabricantes del equipo BTS ${ }^{\circledast}$ (Torres, 2007). El software Biomec ${ }^{\circledR}$ se usa para digitalizar la información del movimiento de los marcadores en el espacio y en el tiempo. El software Tracklab ${ }^{\circledast}$ permite observar la figura que se capturó en 3D, la posición y la aceleración de cada uno de los marcadores y a su vez, crear el archivo que se trabaja en el software Smart Analyzer ${ }^{\oplus}$, haciendo una reconstrucción por medio de la manipulación de la figura generada en 3D con los marcadores. Este archivo es el que finalmente sirve para analizar y manipular los cálculos matemáticos sobre la información de los puntos capturados para describir biomecánicamente los gestos deportivos. Entonces, se pueden hacer cálculos espaciales gráficamente sobre puntos, planos, vectores y sistemas de referencia, para visualizar el resultado en un espacio 3D (Torres, 2007).

La captura que se hace en esta investigación tiene dos modos de empleo. El primero se lleva a cabo en tiempo real, en el cual el deportista puede hacer el gesto y el entrenador o el personal interesado en la evaluación del gesto puede observar su realización en el momento de ejecución. El segundo se graba para su posterior tratamiento y edición.

Cada captura de un gesto deportivo requiere un procesamiento en la información, que va a depender de la naturaleza del estudio. Para un análisis preciso, se necesita hacer la reconstrucción de los segmentos corporales con el fin de visibilizar el movimiento de las articulaciones y de los segmentos de manera continua en el tiempo. Estas capturas son muestras discretas en el tiempo realizadas cada $10 \mathrm{~ms}$; por lo tanto, a esta información fragmentada se le debe hacer un proceso de interpolación para generar virtualmente la posición espacial de cualquier marcador en cualquier instante, a fin de garantizar la señal de respuesta. Las señales obtenidas con estos equipos de captura presentan errores comunes al momento de hacer la digitalización, 
pero se corrigen por medio de un suavizado de la señal de movimiento capturada utilizando filtros digitales.

\section{Set de marcadores}

Para hacer las capturas, se utilizaron marcadores reflectivos en el cuerpo del deportista. En cada disciplina deportiva, se debe analizar muy bien el set de marcadores. No existe un protocolo determinado que describa las posiciones anatómicas de los marcadores del futbolista en pateo de balón en movimiento, por lo que se hicieron varias capturas con diferentes modelos para visualizar si hacían falta marcadores en algunas zonas del cuerpo, de manera que se facilitaran los cálculos, y en otras capturas se pudo observar que algunos marcadores se perdían con facilidad.

Finalmente, con estas pruebas se pudo optimizar el modelo del set de marcadores para obtener los cálculos necesarios que caracterizan la técnica $\mathrm{y}$, en lo posible, evitar la pérdida de marcadores.

Para la captura deportiva, se situaron 17 marcadores ubicados sobre segmentos óseos. El modelo con los marcadores completos para el estudio de la técnica deportiva se muestra en la figura 1.

Figura 1. Set de marcadores para evaluar la técnica del fútbol sala
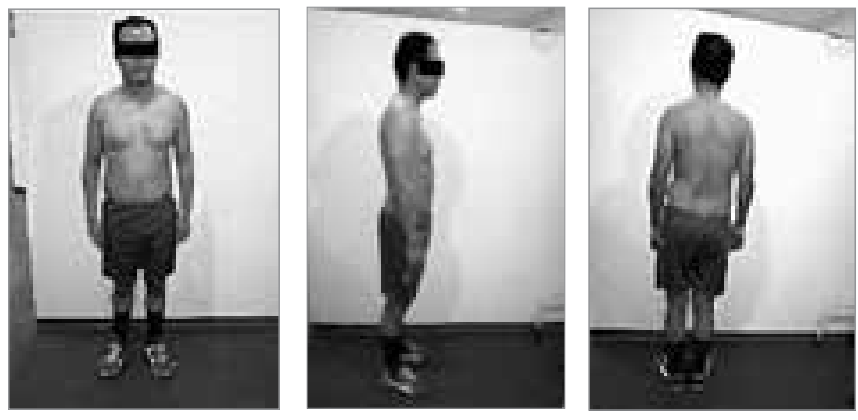

Fuente: elaboración propia.

Las posiciones anatómicas tenidas en cuenta para las capturas de cada uno de los gestos del fútbol sala fueron: 1) hueso frontal; 2) vértebra C7; 3,4) centro articular 
del hombro (tubérculo mayor del húmero izquierdo y derecho); 5,6) articular del codo (epicóndilo externo del húmero derecho e izquierdo); 7,8) extremo articular radial (apófisis esteloide radial derecha e izquierda); 9) sacro; 10,11) Centro articular de la cadera (trocante mayor del fémur derecho e izquierdo; 12,13) centro articular de la rodilla (cóndilo externo del fémur derecho e izquierdo; 14,15) centro articular del tobillo (maléolo externo peroneo derecho e izquierdo) 16,17) quinta falange metatarsiana derecha e izquierda.

Una vez hecha la captura del set de marcadores, por medio del software Tracklab $^{\circledast}$ se pudieron observar los puntos capturados en 3D de la superficie corporal del deportista. A su vez, se puede apreciar, con una interfaz gráfica, el comportamiento de cada uno de los puntos en los ejes $x, y, z$ (eje x: movimientos de progresión y regresión, eje $y$ : movimientos ascendentes y descendentes, y eje $z$ : movimientos laterales), a fin de evaluar comportamientos del marcador como posición en el tiempo y velocidad (Torres, 2007).

Figura 2. Modelo 2D reconstruido del gesto de fondo con el software Tracklab $^{\circledR}$

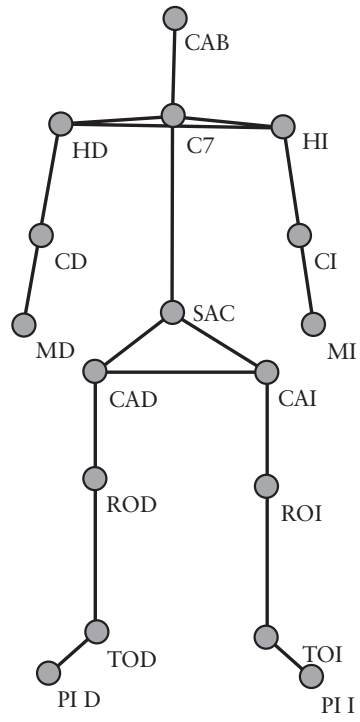

Fuente: elaboración propia.

El modelo reconstruido y que se visualiza en el software Smart Analyzer ${ }^{\circledR}$ para hacer el análisis se evidencia en la figura 3. 
Figura 3. Imágenes dadas por el programa Smart Analyzer ${ }^{\circledR}$
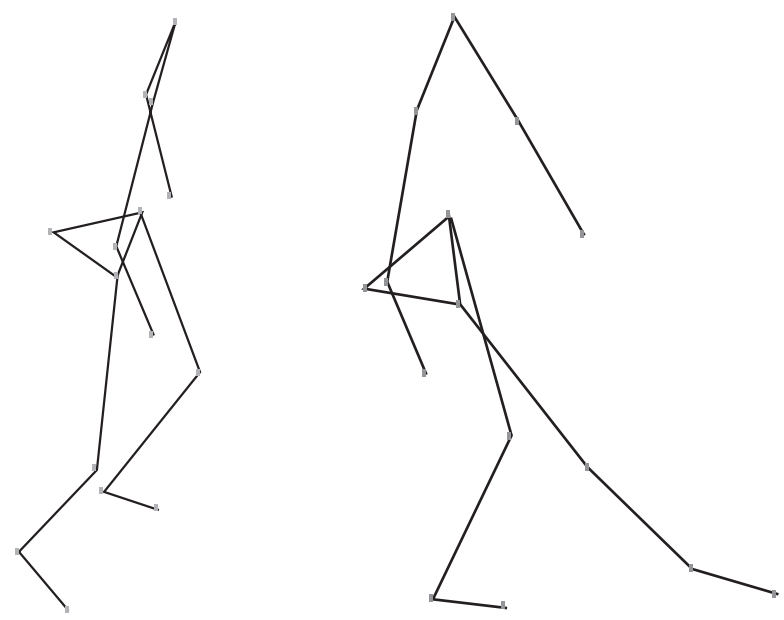

Fuente: elaboración propia.

\section{Deportista}

Se inició el trabajo de investigación indagando en los diferentes centros deportivos de alto rendimiento de fútbol sala para invidentes, de donde finalmente se escogió un deportista, a quien se le hizo una evaluación fisioterapéutica que tuvo en cuenta datos demográficos, historia natural de la enfermedad, y antecedentes patológicos, personales, familiares y deportivos. En la revisión por sistemas, en el sistema osteomuscular se tomó goniometría, adipometría, perímetros, trofismo, postura, entre otros; luego, en el laboratorio de Biomed $^{\circledR}$, se hizo el análisis de la fase de velocidad del lanzamiento de balón quieto y en movimiento para establecer los diferentes movimientos angulares en el lanzamiento de balón.

\section{Resultados}

Los resultados se presentan en dos partes: la primera hace referencia a los resultados de la evaluación fisioterapéutica y la segunda, a lo observado en el laboratorio de biomecánica. 
Se encontró que el deportista escogido, de 23 años, pertenece a la liga de Cundinamarca hace cuatro años, y ha representado a Colombia en diferentes eventos deportivos a nivel nacional e internacional. En la revisión de antecedentes, el atleta refirió el diagnóstico médico de retinitis pigmentosa ${ }^{1}$ a los 2 años de edad; como antecedentes traumatológicos, refirió esguince de cuello de pie izquierdo por accidente de tránsito; a nivel familiar informó que el padre sufre de hipertensión arterial; no reportó ningún otro antecedente patológico, personal, familiar, quirúrgico o deportivo.

En la valoración fisioterapéutica se hizo una revisión por sistemas; a nivel osteomuscular midió $1,65 \mathrm{~cm}$ y pesó $66 \mathrm{~kg}$; el índice de masa corporal fue de $24 \mathrm{~kg} / \mathrm{m}$, que se ubica en un IMC normal. En la evaluación de perímetros, se evidenció aumento del trofismo muscular del hemicuerpo derecho (lado dominante) de 1,5 cm, comparativo con el hemicuerpo contralateral; en miembros inferiores, la diferencia es de 0,5 no significativa ya que el deportista refirió que la mayoría de actividades de entrenamiento las hace bilateralmente. Se hizo la toma de los pliegues cutáneos con el adipómetro en bicipital $19 \mathrm{~mm}$, tricipital $28 \mathrm{~mm}$, subscapular $32 \mathrm{~mm}$, pectoral $27 \mathrm{~mm}$, suprailiaco $28 \mathrm{~mm}$, abdominal $26 \mathrm{~mm}$, muslo $26 \mathrm{~mm}$ y pantorrilla $21 \mathrm{~mm}$.

La postura se evaluó en las tres vistas: se observa antepulsión e inclinación lateral de la cabeza, antepulsión de hombros con descendido de hombro derecho, aumento de la cifosis dorsal, abdomen prominente, flanco derecho disminuido, espina iliaca derecha ascendida, pelvis en anteversión, genu valgo izquierdo y pie izquierdo con eversión. Esto puede deberse a las compensaciones musculares que hace el deportista por el deporte o por la discapacidad que tiene.

En la evaluación de arcos de movilidad articular por goniometría se encontraron rangos articulares dentro de los parámetros normales en cuello, codo, muñeca, tronco y rodilla; pero en hombro están disminuidos por retracciones musculares y cuello de pie por lesión de esguince que no permiten los arcos completos de movimiento. En la valoración de fuerza muscular, según escala de Daniel's, se encontró una fuerza generalizada de entre 3 + y 4, siendo más fuerte la musculatura que se encuentra en pierna derecha.

1 Es una enfermedad hereditaria de los ojos, asociada con degeneración retiniana, que inicialmente afecta más los bastones fotorreceptores. La característica más típica es la reducción de la visión nocturna y periférica en etapas precoces, que suele progresar a ceguera total (Thompson y Nussbaum, 2004). 
En medida real y aparente de miembro superior e inferior, se encuentra una diferencia de $2 \mathrm{~cm}$ en miembro inferior derecho respecto al izquierdo, y de $1 \mathrm{~cm}$ en miembro superior derecho respecto al izquierdo, debido a los imbalances musculares que presenta el deportista, acompañado de las compensaciones y adaptaciones musculares que realiza para efectuar sus gestos deportivos. Presentó retracción en pectoral, cuádriceps, isquiotibiales, psoas y tensor de la fascia lata.

En el patrón de marcha, el deportista hizo contacto, soporte medio, despegue de los dedos, con una longitud de paso de $60 \mathrm{~cm}$ y un ancho de paso de $15 \mathrm{~cm}$; no se evidenció discriminación de cintura escapular.

Los resultados del análisis de las imágenes dadas en el programa Smart Analyzer ${ }^{\circledR}$ se presentan a continuación. Las gráficas arrojadas por el Biomed $^{\circledast}$ muestran la extensión y la flexión máximas del miembro inferior derecho e izquierdo durante la fase de inicio, y de impulsión en posición retrasada y en posición adelantada, durante todo el pateo con balón quieto.

Para analizar la fase inicial, la fase de impulsión en posición retrasada y la fase de impulsión en posición adelantada durante el pateo con balón quieto, se tomaron en cuenta bibliografías que hacen referencia a la biomecánica, que es una ciencia que pretende estudiar los efectos de la energía y las fuerzas de los sistemas biológicos mediante la aplicación de las leyes de Newton en la mecánica de los seres vivos. De esta manera, se hace referencia a la segunda ley, o principio fundamental de la dinámica, según la cual para que un cuerpo altere su movimiento, es necesario que exista algo que provoque dicho cambio. Ese algo es lo que conocemos como fuerza, a menudo denominada impulso (MacDonald, 1989). Si la fuerza total que actúa sobre un cuerpo es nula, la cantidad de movimiento del cuerpo permanece constante en el tiempo; en este caso, la fuerza es la que tendrá el deportista en el momento del pateo y la masa es la medición de la cantidad de materia representada por el balón. El tiempo es la duración de la impulsión de la pierna que hace el golpeo desde una posición retrasada con respecto al tronco hasta una posición adelantada. La mecánica del golpeo del balón conlleva la utilización de una cadena cinética abierta implicada en el pie, la cual suele corresponder, y tiene cierta similitud, con el modelo de marcha o carrera. Aunque se evidencian algunas diferencias, la más notable es la participación del miembro que está en apoyo, el cual desacelera y estabiliza la cadera impidiendo que el cuerpo se desplace hacia adelante. 
La extensión de cadera derecha el deportista la hizo de forma adecuada y presentó -durante la fase de impulsión retrasada- $16^{\circ}$ de extensión en un tiempo de 1,370 segundos, cuando según los parámetros normales, es de $0^{\circ}-20^{\circ}$ de extensión (Suárez, s. f.) (figura 4).

Figura 4. Flexo-extensión cadera derecha

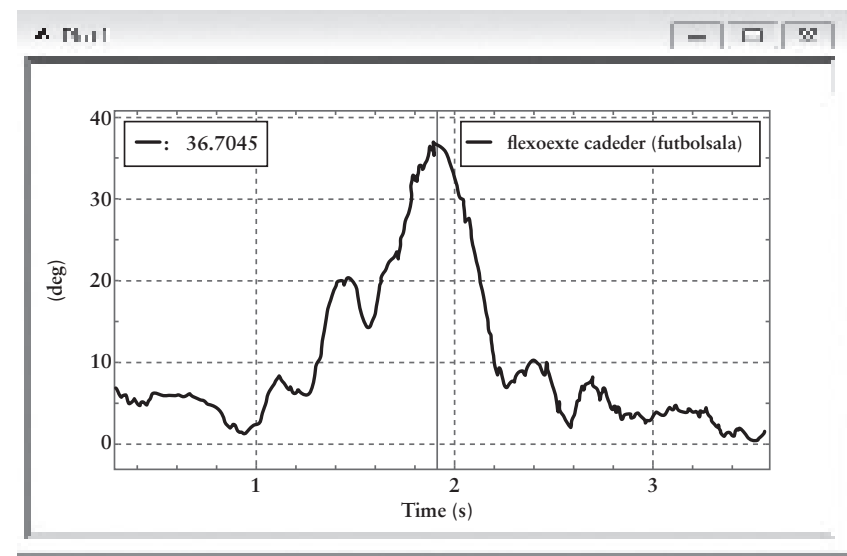

Fuente: elaboración propia.

La flexión de rodilla referida en la literatura (Entrenamientos.org, 2005) es de $0^{\circ}-110^{\circ}$ y de $110^{\circ}-0^{\circ}$ de extensión. El deportista obtuvo una flexoextensión de rodilla derecha, durante la fase inicial, de $14^{\circ}$ de flexión en un tiempo de 0,0 segundos; durante la fase de impulsión retrasada, fue de $15^{\circ}$ de flexión en un tiempo de 1,370 segundos; y en la fase de impulsión adelantada, fue de $7^{\circ}$ de extensión en un tiempo de 1,900 segundos. Esto muestra que el movimiento del deportista se da en un rango mucho menor al referido en un deportista sin discapacidad (figura 5). 
Figura 5. flexo-extensión rodilla derecha

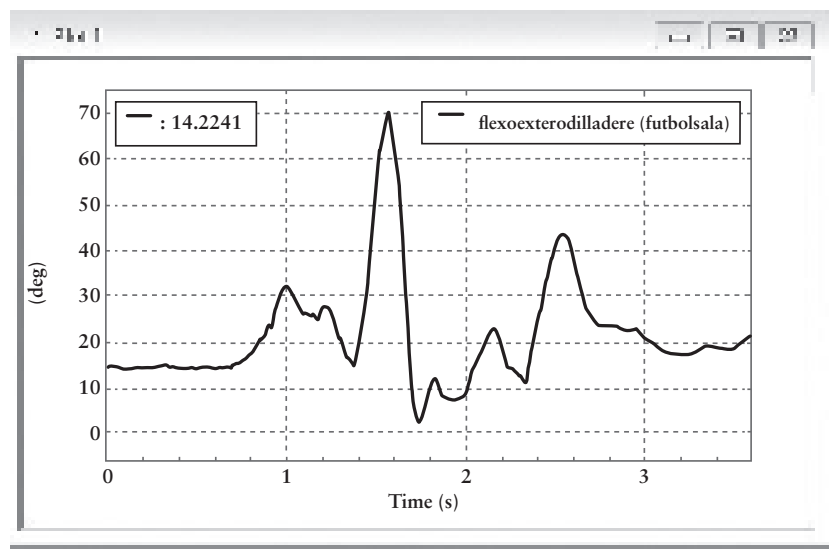

Fuente: elaboración propia.

En el deportista, los movimientos en el tobillo derecho durante la fase inicial fueron de $5^{\circ}$ de plantiflexión cuando inició el movimiento; durante la fase de impulsión retrasada, fueron de $10^{\circ}$ de plantiflexión en un tiempo de 1,370 segundos; y en la fase de impulsión adelantada, fueron de $27^{\circ}$ en un tiempo de 1,900 segundos que dura la acción de esta articulación (figura 6).

Figura 6. Dorsiflexión y plantiflexión de tobillo derecho

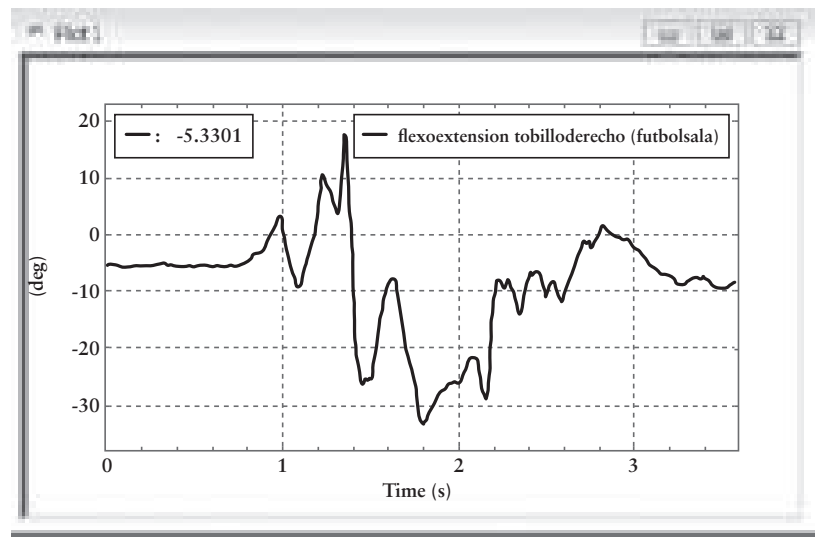

Fuente: elaboración propia. 
Otra de las variables que se tuvieron en cuenta del gesto deportivo fue la de centro de gravedad, la cual se halló a través de la siguiente ecuación (MacDonald, 1989):

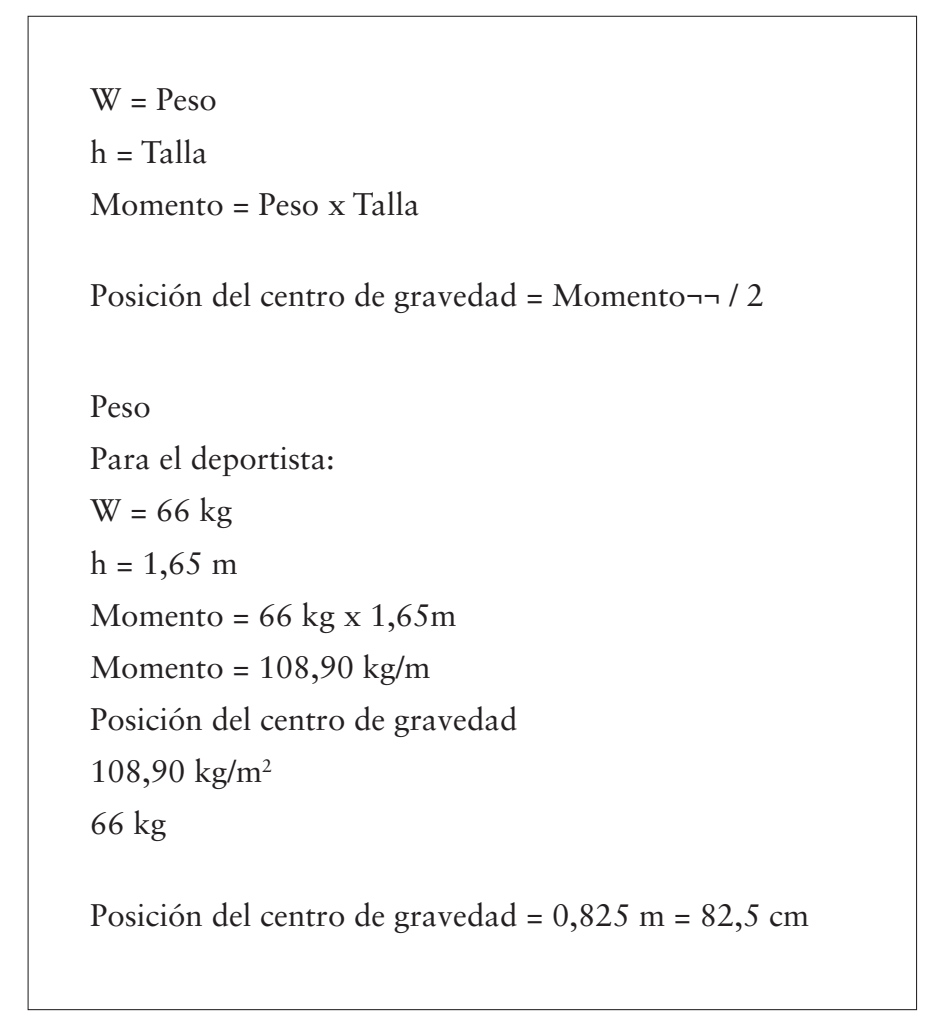

La aplicación de la fórmula para determinar el centro de gravedad indica que se encuentra a los $82,5 \mathrm{~cm}$, es decir que está lateralizado más hacia el lado derecho y más arriba de lo que se describe en la teoría (Rojo, 2006), lo que implica que el hemicuerpo derecho da estabilidad, soporte y fuerza, y el izquierdo es el que brinda velocidad. Esta posición se modifica según el gesto deportivo que se esté desarrollando, con lo que se concluye que se desplaza dependiendo de la acción realizada durante la práctica competitiva, pero este tiende a ir hacia la izquierda y hacia adelante para ayudar a dar potencia al movimiento. 
Figura 7. Centro de gravedad

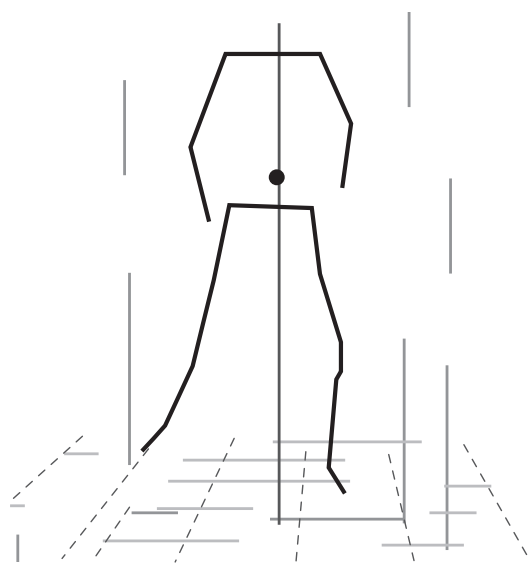

Fuente: elaboración propia.

Para hallar la velocidad de flexo-extensión de rodilla derecha en la fase de inicio, de impulsión en posición retrasada y en posición adelantada durante el pateo con balón quieto, se hizo un cálculo matemático reemplazando la siguiente ecuación (Rojo, 2006):

(Fase 1: Ø reposo), (Fase 2: $\mathrm{m} 2-\mathrm{m} 1 / \mathrm{t} 2-\mathrm{t} 1)$ (Fase 3: $\mathrm{m} 3-\mathrm{m} 2 / \mathrm{t} 3-\mathrm{t} 2)$

En la fase 1 de inicio, tiene una velocidad de $0 \mathrm{~m} / \mathrm{s}$; en la fase 2 de impulsión en posición retrasada, es de $0,2065 \mathrm{~m} / \mathrm{s}$; y en la fase 3 de impulsión en posición adelantada, es de 1,1228 m/s (ver figura 8).

Figura 8. Velocidad

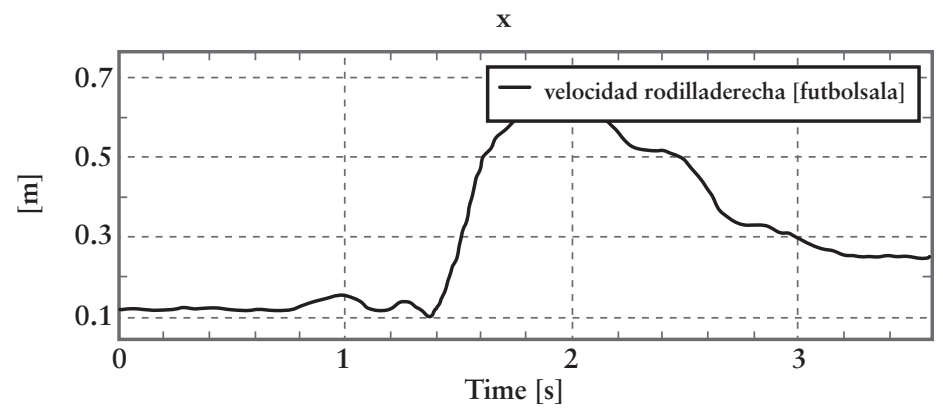




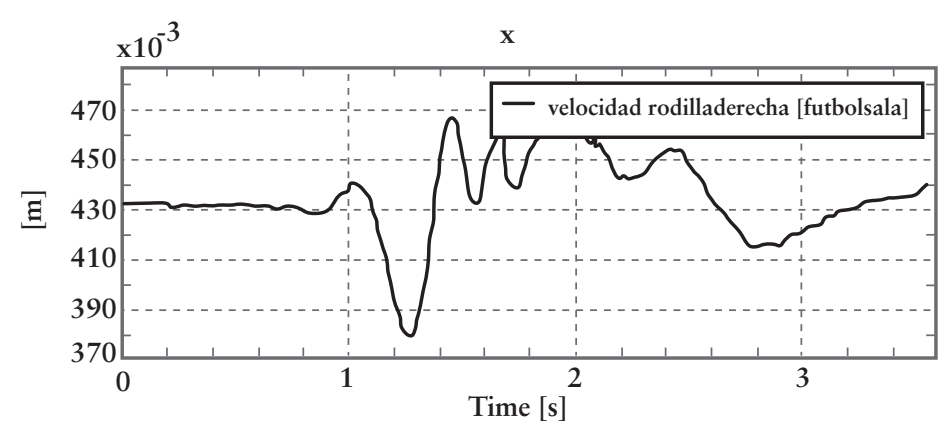

Fuente: elaboración propia.

\section{Discusión}

El fútbol es el deporte más popular y no es ajeno a los deportistas con discapacidad visual, ya que su historia de más de medio siglo avala su práctica a nivel mundial (Urzanqui, 2007; Urmente, 2010). Los resultados de este estudio muestran que el deportista no estaba haciendo de forma adecuada el gesto deportivo, al presentar disminución de arcos de movilidad en la fase de pateo con balón quieto, lo que implica una reducción de la potencia y la velocidad del gesto. El movimiento realizado influye negativamente en el rendimiento deportivo, pues presenta una mala alineación corporal, provocando la disminución de los rangos de movimiento articular, especialmente en los miembros inferiores utilizados en la práctica deportiva (González, 2008).

El deportista en condición de discapacidad visual presentó una flexión de cadera por debajo del rango normal, resultado que posiblemente se da por los desequilibrios posturales que adopta y que afectan, sin duda, la disposición de los segmentos óseos por el desplazamiento del centro de gravedad, que provoca alteraciones en los músculos que movilizan la pelvis (Lomas, 2006).

Se analizó el desplazamiento articular de miembro inferior, con lo que se evidenció que el deportista hace una mayor flexión de cadera derecha para hacer el pateo. Aunque según la bibliografía la flexión máxima es de $120^{\circ}$, en este movimiento no hay necesidad de hacer flexión máxima, pero sí mayor a lo mostrado, porque esto le brinda al movimiento potencia y velocidad. También, se evidenció que durante la fase de impulsión, tanto en posición retrasada como en posición adelantada de la rodilla izquierda, el deportista no extiende la rodilla, ya que su centro de gravedad está muy adelante de su cuerpo y esto impide el movimiento. 
La flexión de la rodilla derecha es menor, comparada con la izquierda, en el momento de hacer la fase de impulsión en posición atrasada, con la cual el deportista hace el pateo. Esta diferencia se debe a las retracciones que presenta a nivel de cuádriceps, que genera disminución de la potencia de la patada hacia el balón.

En cuanto a la articulación de cuello de pie, se evidencian rangos de movimiento amplios, de acuerdo con los parámetros normales referidos en la bibliografía. Pero dichos movimientos posiblemente son producidos por la hiperlaxitud, causada por el esguince ya descrito en la evaluación fisioterapéutica, los cuales generan inestabilidad durante el pateo.

Respecto al movimiento del centro de gravedad, se contempla teóricamente cómo es el punto en el que la masa de todos los segmentos corporales se distribuye de manera homogénea. En posición anatómica, se considera que el centro de gravedad se sitúa a la altura de la segunda vértebra sacra, en el interior de la pelvis; sin embargo, tan pronto como la configuración del cuerpo es distinta de la posición anatómica (Rojo, 2006), el centro de gravedad se desplaza, e incluso puede llegar a localizarse en el exterior del cuerpo cuando se produce un movimiento definido como una fuente de desequilibrio, puesto que combina la geometría del cuerpo que modifica la posición del centro de gravedad. Se evidenció que el centro de gravedad del deportista, al estar lateralizado por el desequilibrio muscular que presentó, afectó la efectividad del movimiento.

Cuando la pelvis está en apoyo unilateral, el equilibrio transversal se asegura únicamente mediante la acción de los abductores del lado de apoyo; dado el peso del cuerpo aplicado al centro de gravedad, la pelvis tiende a bascular en torno a la cadera que carga. En este caso, se puede considerar la cadera como una palanca de segundo grado, cuyo punto de apoyo está constituido por: la cadera que carga, la resistencia por el peso del cuerpo y la potencia por la fuerza del glúteo medio aplicada a la fosa iliaca anterosuperior, como accesorio de los glúteos, también en el apoyo unilateral de la cadera cuentan con la ayuda del tensor de la fascia lata.

En el sistema nervioso, intervienen sinergias cinemáticas para compensar con precisión el desplazamiento del centro de gravedad (Viel, 2002). Además, se encuentran mecanismos articulares en la ejecución de los movimientos, en los que influyen movimientos sincrónicos entre miembro superior e inferior que dan como resultado un desplazamiento sinuidal del 
centro de gravedad. Estos mecanismos que se producen son los responsables de que el desplazamiento del centro de gravedad se haga sin cambios bruscos de dirección, lo que supone un ahorro energético para el organismo. Teniendo en cuenta los aspectos anteriores, podemos decir que el desplazamiento del centro de gravedad se ve afectado por alteraciones posturales del lado derecho, razón por la cual tiende a ubicarse sobre este lado, para generar su estabilización y disminuir la fricción dada por compensaciones biomecánicas del lado izquierdo.

Por otra parte, al aplicar la fórmula para la determinación de la velocidad media en cualquier evento, se debe dividir el desplazamiento entre el tiempo utilizado para ejecutar la acción evaluada (MacDonald, 1989; Pérez y Braso, 2002). Al interpretar la fórmula para la velocidad del movimiento de flexo-extensión en la rodilla derecha, se observó que fue el segmento más veloz, puesto que en la fase de impulsión en posición retrasada se obtuvo $0,2065 \mathrm{~m} / \mathrm{s}$ y en la fase de impulsión en posición adelantada fue de 1,128 $\mathrm{m} / \mathrm{s}$ respecto al cuello de pie derecho. En este segmento corporal, según la ecuación aplicada, se obtuvieron los siguientes resultados: $0,1593 \mathrm{~m} / \mathrm{s}$ en la fase de impulsión en posición retrasada y $0,53 \mathrm{~m} / \mathrm{s}$ en la fase de impulsión en posición adelantada.

La velocidad de la marcha en personas sin condición de discapacidad se puede incrementar hasta en $44 \%$; sin embargo, las personas con algún cambio en el ciclo de la marcha, como se observó en este deportista, presentan problemas en los mecanismos de amortiguación (Daza, 2007), lo que genera que se vea afectada la dispersión de la energía cinética por almacenamiento como energía potencial al iniciar el movimiento.

Si se considera el sistema aislado, representado por el pie que se mueve hacia adelante y el balón firme sobre el terreno de juego, el balón depende del momento inercial del miembro inferior y de la velocidad lineal del pie, que es la consecuencia de los momentos de fuerza generados por los músculos que actúan a nivel de la cadera, la rodilla y el tobillo (Mognoni y Sirtori, 1996).

Los estudios más recientes muestran que al provocar aumentos de torque de cuádriceps del orden de $20 \%$, se obtiene aumento de la velocidad máxima del balón del 1-2 \%, lo que no es significativo desde el punto de vista estadístico, pero podría ser una potencial recomendación para mejorar el gesto deportivo. Sin embargo, los autores sostienen que la fuerza sería mayor si se entrenaran los músculos flexores del muslo (Mognoni y Sirtori, 1996). 
La potencia que se maneja en el movimiento del deportista en la rodilla derecha sería mayor si se disminuyeran las retracciones musculares de cuádriceps e isquiotibiales que están evidenciadas en la evaluación, puesto que dichas retracciones evitan una mayor potencia y fuerza al hacer el pateo.

\section{Conclusiones}

Por medio de esta investigación, se puede caracterizar la técnica del deportista de una forma cuantitativa, utilizando una herramienta de alta tecnología, en la se hace un análisis de cada una de las técnicas competitivas utilizadas en los diferentes deportes.

En este estudio, se determinó que el deportista no estaba haciendo de forma adecuada el gesto deportivo, porque no alcanzaba los arcos de movimiento normales durante la fase de pateo con balón quieto. El movimiento realizado influye negativamente en el rendimiento deportivo, lo cual ocasiona una mala alineación corporal y provoca la disminución de los rangos de movimiento impacto. La cantidad de movimiento total está dada por el producto entre la velocidad del pie y la masa inercial de miembro inferior (Mognoni y Sirtori, 1996); y como la segunda masa es inferior a la primera, el balón adquiere una velocidad que es superior cerca del $30 \%$ con respecto a la máxima velocidad del pie, inmediatamente al primer contacto.

En futuros estudios, se espera ampliar el tamaño de la muestra para poder comparar las diferentes técnicas en cada una de las modalidades deportivas, con miras a establecer la caracterización de la disciplina. De igual forma, se puede aplicar a las diferentes disciplinas deportivas, a fin de hacer un análisis adecuado del gesto deportivo y mejorar las técnicas y el rendimiento de los jugadores.

Con respecto a la ejecución de la técnica, se recomienda al entrenador y al deportista paralímpico incluir en sus programas de entrenamiento ejercicios de flexibilidad en miembros superiores, inferiores y tronco para poder mejorar las compensaciones musculares generadas por la práctica deportiva y su discapacidad.

A su vez, se espera fortalecer el hemicuerpo izquierdo, por el imbalance muscular existente, para mejorar la distribución del manejo de fuerzas en el momento del pateo y así no sobrecargar ninguna de las articulaciones ni generar contrapesos que puedan, con el tiempo, producir lesiones de orden muscular, ligamentario u óseo. 


\section{Referencias}

Campos López, C. (2010). Fútbol sala. Federación Española de Deportes para Ciegos (FEDC). Recuperado el 15 de abril de 2013, de www.fedc.es/appdocumentos/ fedec/prod/05_Futbolsala.doc

Castro Jiménez, L. (2010). Caracterización biomecánica con tecnología digital en el deporte para personas en condición de discapacidad: una revisión sistemática. Umbral Científico, (17), 31-39.

Daza, J. (2007). Evaluación clínico-funcional del movimiento corporal humano. Madrid: Editorial Médica Panamericana.

Entrenamientos.org (16 de junio de 2005). Entrenamiento fútbol: aspectos biomecánicos del rendimiento en el fútbol, control de movimientos de habilidad. Recuperado de http://www.entrenamientos.org/Article2.html

González Figueiras, O. (2008). Análisis de las lesiones en el fútbol sala popular. Efdeportes Revista Digital, 13(120). Recuperado de http://www.efdeportes. com/efd120/analisis-de-las-lesiones-en-el-futbol-sala-popular.htm

Lomas, M. (2006). Estabilidad y centro de gravedad. Recuperado de http://www. mailxmail.com/curso-ejercicio-fisico/estabilidad-centro-gravedad.

MacDonald, S. (1989). Física para las ciencias de la vida y de la salud. Wilmington: Adison Wesley Iberoamericana.

Mognoni, P. y Sirtori, M. (1996). La fuerza muscular del futbolista. Demanda y respuesta sobre la fuerza en el jugador de fútbol. Revista de Actualización en Ciencias del Deporte, 4(13).

Molina, P. y Montenegro, Y. (2006). Análisis del gesto deportivo en invidentes que practican fútbol sala. Bogotá: Universidad Manuela Beltrán.

Pérez, M. y Braso, J. (2002). La velocidad en el fútbol sala. Manifestaciones de la velocidad. Efdeportes Revista Digital, 8(47). Recuperado de http://www.efdeportes.com/efd47/fsala.htm

Rojo, J. (2006). Fundamentos del movimiento humano. Bogotá: Elsevier Masson.

Secretaría de Cultura, Recreación y Deporte. (2003). Deporte paralímpico. Recuperado el 15 de abril de 2013, de http://culturarecreacionydeporte.gov.co/ portal/nodel/203

Suárez, Y. (s. f.). Aspectos técnicos fundamentales en el fútbol sala: fundamentos técnicos con balón. Recuperado de http://wikialbon.wikispaces.com/file/view/ tecnica_fundamentos.pdf

Thompson, M. y Nussbaum, J. (2004). Genética en medicina (5. ${ }^{a}$ ed.). Barcelona: Elsevier Masson. 
Torres, A. (2007). Análisis biomecánico del esgrima mediante sistemas optoelectrónicos de análisis de movimiento. Estudio de caso: deportista de alto rendmiento. Revista Ingeniería Biomédica, (2), 30-39.

Urmente, J. M. (2010). Teoría del entrenamiento del fútbol-sala, principios ofensivos. Recuperado de http://www.mailxmail.com/curso-teoria-entrenamientofutbol-sala/principios-ofensivos-1-ataque-contraataque-apoyos-rotaciones

Urzanqui Velasco, A. (2007). Beneficios del ejercicio físico y el deporte para la salud de los ciegos y deficientes visuales. En: Urzanqui Velasco, A. (Comp.), Segunda Conferencia Internacional sobre Deporte Adaptado. Málaga: Fundación Andalucía Olímpica.

Viel, E. (2002). La marcha humana: biomecánica, exploraciones, normas y alteraciones. Barcelona: Elsevier Masson. 